\title{
Research on One-Piece Structure Target Flow Sensing Technology Based on Fiber Bragg Grating
}

\author{
Chuntong LIU, Zhengyi ZHANG*, Hongcai LI, Zhenxin HE, \\ Xiaofeng ZHAO, and Rongjing WU
}

Department Two, Rocket Force University of Engineering, Xi'an 710025, China
${ }^{*}$ Corresponding author: Zhengyi ZHANG $\quad$ E-mail: 13022719922@163.com

\begin{abstract}
In view of problems existing in the detection of the traditional hydraulic system, such as the large volume of sensor and the low measurement accuracy, a new one-piece target type flow sensor is designed and researched based on fiber Bragg grating (FBG). A compact structure is designed, which is convenient to be dismantled, processed, and installed, based on the analysis of the principle of FBG and the structure of target type flow sensor. The force of target put in fluid flow is turned into the FBG wavelength drift, with a corresponding relationship. The problem on the cross sensitivities of the temperature and strain is solved effectively by using double FBG symmetrically pasted on the both surfaces of the cantilever. The impact on the fluid state is analyzed through simulation in the software FLUENT, and the results show that the impact was smaller than that of the traditional structure. The results of experiments in the hydraulic system show that there is a good linear relationship between the change in the dual FBG central wavelength and mass loading on the target sheet has a good linear relationship, and the sensitivity is twice that of a single FBG sensitivity.
\end{abstract}

Keywords: Fiber Bragg grating; flow sensor; target type structure; equal intensity cantilever beam

Citation: Chuntong LIU, Zhengyi ZHANG, Hongcai LI, Zhenxin HE, Xiaofeng ZHAO, and Rongjing WU, "Research on One-Piece Structure Target Flow Sensing Technology Based on Fiber Bragg Grating," Photonic Sensors, 2016, 6(4): 303-311.

\section{Introduction}

In the industrial production process and automation control field, the flow is one of the parameters being detected and controlled. Flow meter is also used in the detection of widely used varied and mechanical rotor flow sensor [1]. But due to the complexity of the mechanical structure, its measurement accuracy is low. Ultrasonic flowmeter [2], the electromagnetic flowmeter [3], and acoustics Doppler current meter [4] have a high measurement accuracy, and the operation is simple, but their cost is high, and they are interferenced easily by electromagnetic. Fiber Bragg grating (FBG) flow sensor is a new type of sensing technology developed in recent 20 years $[5,6]$, and its principle is that the central Bragg reflected wavelength is sensitive to physical quantities of the external physics, through the special package and structure design to realize measurement of various parameters [7, 8]. Compared with the traditional electrical sensors, FBG has lots of advantages, such as small volume, light weight, high sensitivity, the absolute measurement, and strong ability of resistance against electromagnetic interference. It combines sensing and transmission, and it is easy to be reused and constitutes a sensor network $[9,10]$. Therefore, it's very suitable for a hydraulic system in detection of

Received: 25 May 2016 / Revised: 17 July 2016

(C) The Author(s) 2016. This article is published with open access at Springerlink.com

DOI: $10.1007 / \mathrm{s} 13320-016-0352-6$

Article type: Regular 
flow parameters, such as high precision and multiple point detection requirements.

In recent years, Yu et al. [11], Wang et al. [12], Wang et al. [13], and others have applied FBG in the traditional target flowmeter and have realized the accurate and quick measurement of flow by FBG based on the circular target and a cantilever beam structure, but its structure is complex and not easy to be installed and sealed, and it fails to give full play to the advantages of FBG sensor. According to the above background, this paper proposes a set of choke target slices and sensitive elements in one of equal strength cantilever structures, on the basis of analyzing the measuring principle, and after the structure design and material processing, the feasibility of this structure is verified by experiment, and the sensor is proved sensitive and reliable.

\section{Basic principle}

\subsection{Basic principle of FBG sensing}

According to coupled-mode theory, for broadband incident light, the refractive index of FBG cycle disturbances affects only a very narrow range of wavelengths of spectral period of impact, that is, only to meet the Bragg condition

$$
\lambda_{B}=2 n_{\text {eff }} \Lambda
$$

where $\lambda_{B}$ is the center of Bragg reflection wavelength, $n_{\text {eff }}$ is the effective refractive index, and $\Lambda$ is the grating period.

Light waves can be reflected by grating, the transmission spectra of the rest are not affected, and FBG will play the role of reflector or filter. Equation (1) shows that $\lambda_{B}$ changes with $\Lambda$ and $n_{\text {eff }}$. Temperature and strain are the physical parameters which FBG is directly sensitive to, and they can cause $\Lambda$ and $n_{\text {eff }}$ to change. FBG is directly sensitive to the strain and temperature, and they will also change $\Lambda$ and $n_{\text {eff. }} \lambda_{B}$ is influenced by temperature through elastic-optic effect and thermal expansion effect, and $\lambda_{B}$ is influenced by strain through elastic-optic effect and the grating period. The drifting of $\lambda_{B}$ caused by strain and temperature can be deduced as

$$
\Delta \lambda_{B}=(\alpha+\xi) \lambda_{B} \Delta T+\left(1-P_{e}\right) \lambda_{B} \varepsilon=K_{T} \Delta T+K_{\varepsilon} \varepsilon
$$

where $\alpha$ and $\xi$ are the thermal expansion coefficient and thermal optical coefficient of the fiber respectively; $P_{e}$ is the effective elastic-optic coefficient; $\varepsilon$ is the axial strain; $K_{T}$ and $K_{\varepsilon}$ are the sensitivity coefficients of temperature and strain. Equation (2) is the theoretical basis of analyzing FBG sensing process. When there is outside temperature and stress field on FBG, the appropriate information can be accurately obtained accurately by measuring the change in the reflection wavelength with the wavelength demodulation, which is the basic principle of FBG sensing.

\subsection{Target type flow sensing principle of FBG}

According to the basic principle of FBG sensing, FBG is sensitive to temperature and strain directly, so to realize the precise measurement to flow, the special structure should be designed to turn flow into the physical quantity to which FBG is sensitive.

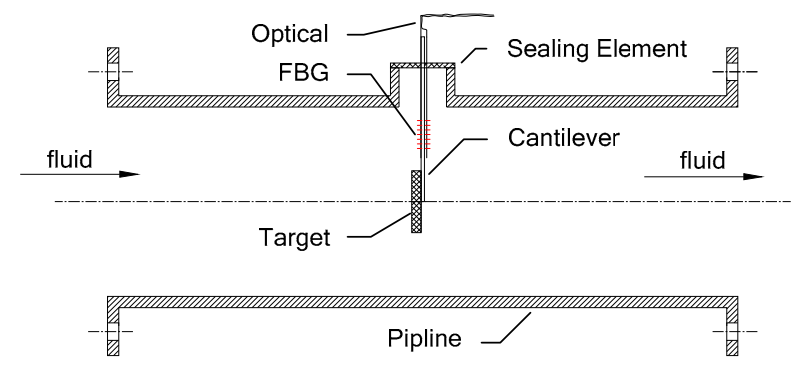

Fig. 1 Target type flow sensing principle diagram.

In the paper, the structure of the target type flow measurement is used, and the structure principle is shown in Fig. 1. At the axis of the pipeline which the fluid flows through, a disc-shaped target sheet is placed, and an annular space is formed. Target piece is fixed on the triangle cantilever beam, and the force from the flow on the target piece is converted into the strain of the triangle cantilever beam which is measured by the sensitive element.

When the target is impacted by fluid, it is subjected to a resultant force which is composed of 
three forces: the fluid dynamic pressure, fluid static pressure (differential pressure), and viscous friction, and the first two forces play a leading role when the flow is large. By the Bernoulli equation, we can derive [15]

$$
A_{1}\left(p_{0}-p\right)+A_{1}\left(\frac{p v_{0}^{2}}{2}-\frac{p v^{2}}{2}\right)=\frac{1}{2} \rho \xi v^{2} A_{1}
$$

where $\rho$ is the fluid density; $A_{1}$ is the target area; $\zeta$ is the local resistance coefficient; $v_{0}$ and $p_{0}$ are the average flow velocity and pressure of fluid in front of the target, respectively; $v$ and $p$ are the average flow velocity and pressure of fluid in behind of the target, respectively; $A_{1}\left(p_{0}-p\right)$ is the static pressure on the target and referred to as $F_{1} ; A_{1}\left(\rho v_{0}^{2} / 2-\rho v^{2} / 2\right)$ is the dynamic pressure on the target and referred to as $F_{2} ; F_{1}+F_{2}$ is the resultant force on the target and referred to as $F$, and $F$ within $v$ is given as

$$
F=\frac{1}{2} \rho \zeta v^{2} A_{1} .
$$

The FBG attached to both sides of the central axis of the triangle cantilever beam is deformed by the force on the triangle cantilever beam caused by the flowing fluid, and the central wavelength of reflection wave is changed. The central wavelength of the grating in the direction of flow drifts in the direction of increasing, the central wavelength of the grating against the direction of flow drifts in the direction of decreasing, and the amount of drift is the same. Because they are influenced by the temperature at the same time, the change in the two FBG wavelength is given as [16]

$$
\begin{aligned}
& \Delta \lambda_{B 1} / \lambda_{B}=\left(1-P_{e}\right) \varepsilon+(\xi+\alpha) \Delta T \\
& \Delta \lambda_{B 2} / \lambda_{B}=-\left(1-P_{e}\right) \varepsilon+(\xi+\alpha) \Delta T .
\end{aligned}
$$

The change in double FBG wavelength $\Delta \lambda$ within the grating axial strain $\varepsilon$ is given as

$$
\Delta \lambda=\Delta \lambda_{B 1}-\Delta \lambda_{B 2}=2 \varepsilon\left(1-P_{\varepsilon}\right) \lambda_{B} .
$$

Equation (7) shows that the sensitivity is improved by the differential structure of double FBG, and at the same time, the problem on the cross sensitivities of the temperature and strain is solved effectively. According to the mechanics of materials, the strain of the triangle cantilever beam $\varepsilon$ within the loading force in the free end $F$ is given as [17]

$$
\varepsilon=\frac{6 F L}{E b h^{2}}
$$

where $b$ is the bottom width of beam; $h$ is the thickness; $E$ is the young's modulus of cantilever beam material; $L$ is the length of the cantilever beam fixed end.

We can discern from (7) and (8) that $\Delta \lambda$ within $F$ is given as

$$
\Delta \lambda=\frac{12 F L}{E b h^{2}}\left(1-P_{e}\right) \lambda_{B} .
$$

Supposing oil in the pipeline is incompressible fluid, according to the continuity equation of the total fluid flow, we can discern from (4), (8), and (9) that the total flow $Q$ in the pipeline can be deduced as

$$
Q=A_{2} v=\left(D^{2}-d^{2}\right) \sqrt{\frac{\pi b h^{2} E}{24\left(1-P_{\varepsilon}\right) L \zeta \rho d^{2}}} \cdot \sqrt{\Delta \lambda}=K \cdot \sqrt{\Delta \lambda}(10)
$$

where $D$ is the pipe diameter; $d$ is the target path; $A_{2}$ is the annulus area between $D$ and $d ; Q$ is the flow through $A_{2}$.

$P_{e}$ of the typical quartz fiber is equal to $0.22, b, h$, $L$, and $E$ are constants if cantilever beam size and target size are determined, and $\rho$ and $\zeta$ are fixed values for a particular fluid. So from (10) we can get that the fluid characteristic parameters under the given conditions can be accurately measured as long as measuring the amount of wavelength change of FBG.

\section{Structure design}

\subsection{One-piece structure design of the target type flow sensor}

The principle of the traditional target flowmeter is that the resistance strain gauges is sensitive to deformation of the target caused by the fluid flow, and the sensitive part of the electrical devices must be isolated from measured media, so the flowmeter is very difficult to be processed, handled, and sealed. And the impact force on the target sheet must be achieved by the force transmission rod. However, 
FBG is non-corrodible and non-electrical component, so in the paper, the force transmission mechanism is omitted, and the target sheet and the triangle cantilever beam are made organically integrate to simplify design.

In Fig. 2, the sensor is mainly composed of four parts of valve body, convex platform, end cover, and one-piece target. Body, convex platform, and end cover are processed with hard aluminum alloy, and one-piece target piece are processed entirely by the method of linear cutting. The center of target sheet must be coincided with the axis of pipeline in the whole installation process, and target sheet is perpendicular to the direction of fluid flow, which relies mainly on the convex part of the convex platform to achieve. Type $\mathrm{O}$ sealing ring is used to face seal between the valve body and convex platform, end cover and convex platform, and the sealing cap is fastened by screws. Two FBGs are pasted on both sides of the central axis of the triangle cantilever beam symmetrical, and the differential configuration is formed. The impact on the target piece from the fluid causes the deformation of the cantilever and FBG posted on both sides, so the size of the flow can be measured according to the offset of the central reflection wavelength.

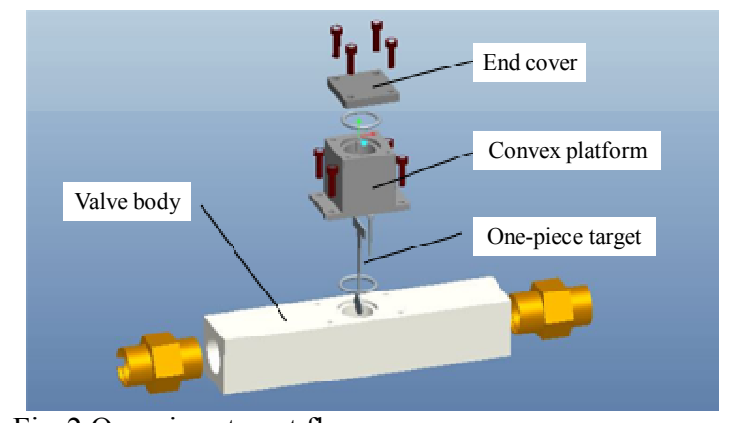

Fig. 2 One-piece target flow sensor.

\subsection{One-piece structure design of the target piece}

The sensitivity and performance of the target type flow sensor mainly depends on the rationality of the structural design of the one-piece target piece. The design principles is as follows:

(1) The size of the ratio of pipe-diameter should be moderate, and the sensitivity and pressure loss should be taken into account.

(2) The measuring range of sensor should be considered when determining the size of the triangle cantilever beam, and the accuracy should meet the requirements within the range.

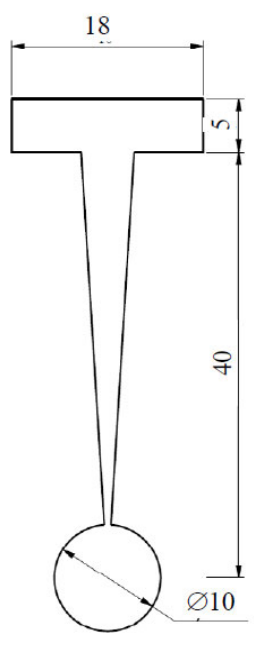

(a)

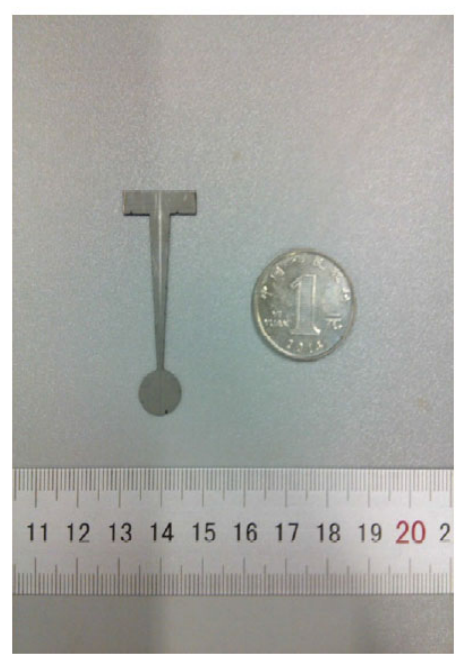

(b)
Fig. 3 One-piece target: (a) processing drawings and (b) material object.

When the pipe diameter is determined, differential pressure gradually increases with an increase in diameter of target piece, and that is to say, the pressure loss through the one-piece target type sensor is larger. Combing with the practical application, we select the body diameter $D$ equal to $16 \mathrm{~mm}$ and the target diameter equal to $10 \mathrm{~mm}$. The range of the flow sensor is designed between $0 \mathrm{~L} / \mathrm{s}$ and $2 \mathrm{~L} / \mathrm{s}$, so the speed of the fluid flowing through the gap between the pipeline and target sheet is at a range of 0 to $16.3 \mathrm{~m} / \mathrm{s}$, and then you can calculate the range of the impact on the one-piece target. The measurement accuracy should be increased as far as possible in the process of designing the one-piece target on the premise that the strength requirements are met. The one-piece target is designed as shown in Fig. 3. The processing drawing is shown in Fig. 3(a), and the material object is shown in Fig. 3(b). The material of the one-piece target is $304 \#$ stainless steel, the thickness is equal to $1 \mathrm{~mm}$, the length of 
the cantilever $l$ is equal to $40 \mathrm{~mm}$, the bottom width $b$ is equal to $5 \mathrm{~mm}$, the diameter of circular target sheet is equal to $10 \mathrm{~mm}$, and the center of the circular target sheet is coincided with the vertex of the triangle cantilever beam. The length of the rectangle area above the target piece is equal to $18 \mathrm{~mm}$, and the width is equal to $5 \mathrm{~mm}$. In the process of installation, it is placed in the grooves of convex platform to fasten the one-piece target and ensure that the target sheet plane is perpendicular to the direction of the fluid flow.

\section{FLUENT simulation analysis}

We use FLUENT software for one-piece target type flow sensor fluid analyzing. Through the simulation experiment, we observe the status of fluid and compare it with that of traditional target type flow sensors, to determine whether the design of one-piece target structure is feasible.

The 3D geometric model is set up in the GAMBIT software, and flow sensor body part is designed to a tee model, of which the pipe diameter is $16 \mathrm{~mm}$, the horizontal line is $150 \mathrm{~mm}$, and vertical pipeline is $40 \mathrm{~mm}$. Oil in a horizontal pipe flows along the $X$-axis direction. Solution 1 is for the structure of one-piece target type, and Solution 2 is for the traditional target type structure, except for the target piece of different structures, other conditions of the two schemes are consistent. Meshing with Tet/Hybrid (the form of tetrahedral mesh is given priority to the appropriate position including hexahedral grid, cone, and wedge) and Tgrid (it will be divided into tetrahedron grid cell body, and in the proper position it can also contain the cone, hexahedron, and wedge unit) type, the grid size is set to 0.5. Target around the grid uses refinement uprocessing by wall partition boundary grid. After meshing, the model diagrams as shown in Figs. 4(a) and 4(b) are one-piece target type structure and the traditional model of the target structure, respectively.
After the model is established in this paper, we export mesh file from GAMBIT, open the FLUENT software, choose three dimensional single precision calculating body, import the mesh file, and check the meshing condition. According to the actual situation, we set the parameters of the segregated solver and implicit solver. The k-epsilion model is chosen as the viscosity model, and the oil is chosen as the fluid. The boundary condition is that the inlet velocity is $10 \mathrm{~m} / \mathrm{s}$, and other conditions are initial settings of FLUENT software.

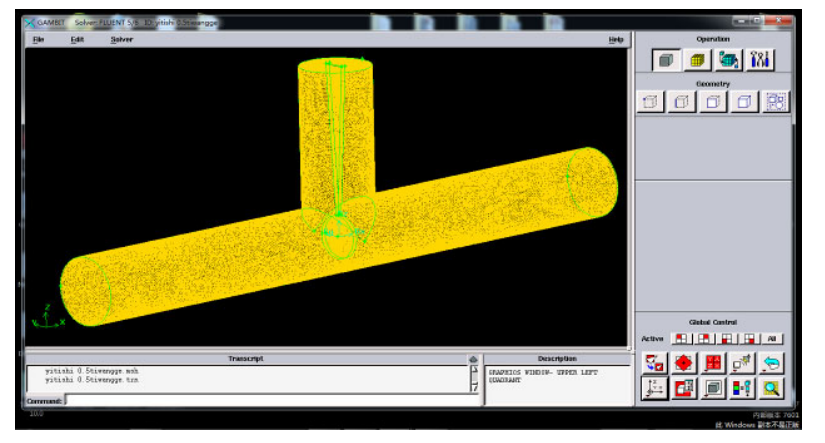

(a)

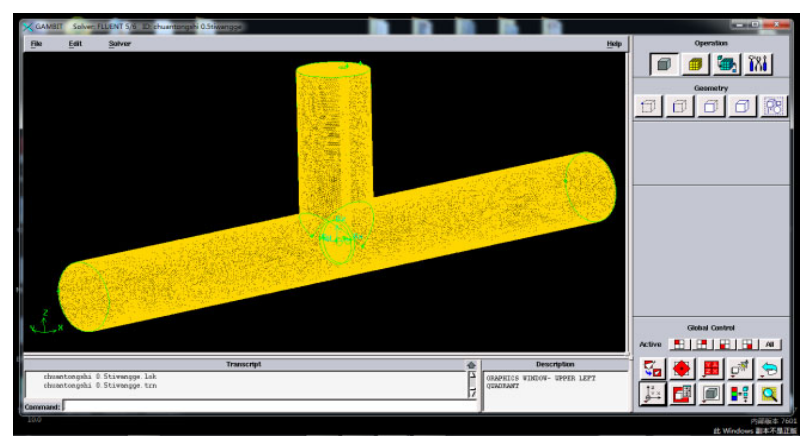

(b)

Fig. 4 One-piece target type flow sensor structure model diagram: (a) one-piece target type structure and (b) traditional model of the target structure.

When solving initialization, the inlet should be selected. The convergence precision $k$ and epsilon are both set to 0.001 . Then start to solve, after solving, set up three sections as $X O Z$ (main view), $X O Y$ (top view), and $Y O Z$ view (left view).

As shown in Fig. 5, there are two kinds of scheme of the top view of the dynamic pressure contours (plane $X O Y$ ). Figure 6 shows the main view of the velocity contours (plane XOZ). Figure 7 shows the top view of the flow chart (plane $X O Y$ ). 


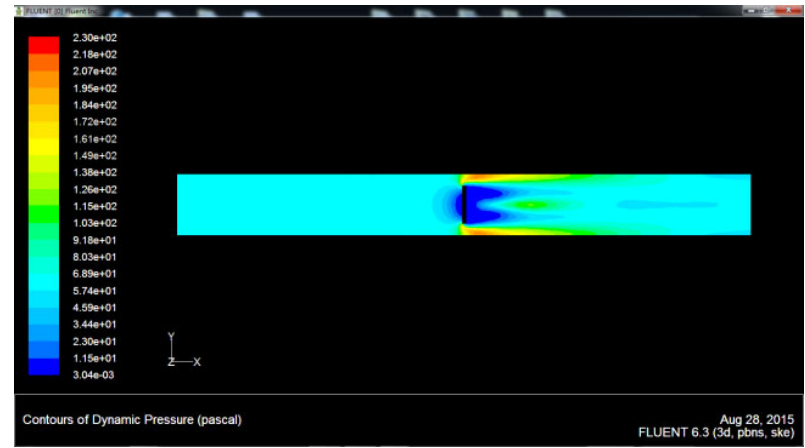

(a)

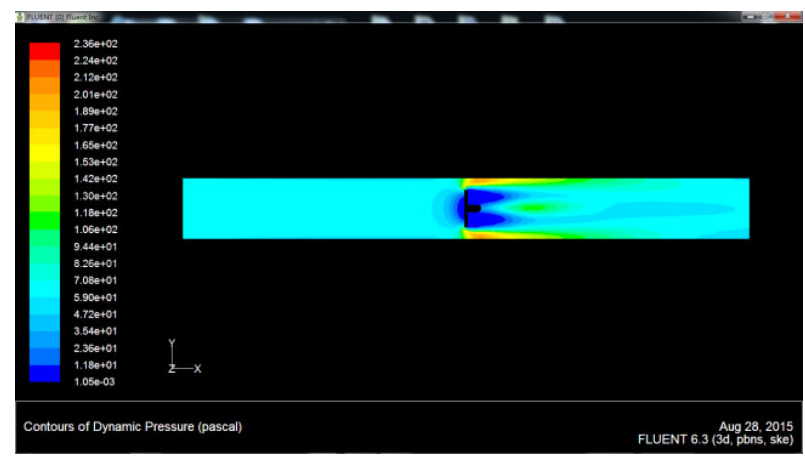

(b)

Fig. 5 Dynamic stress nephogram: (a) one-piece target type structure and (b) traditional model of the target structure.

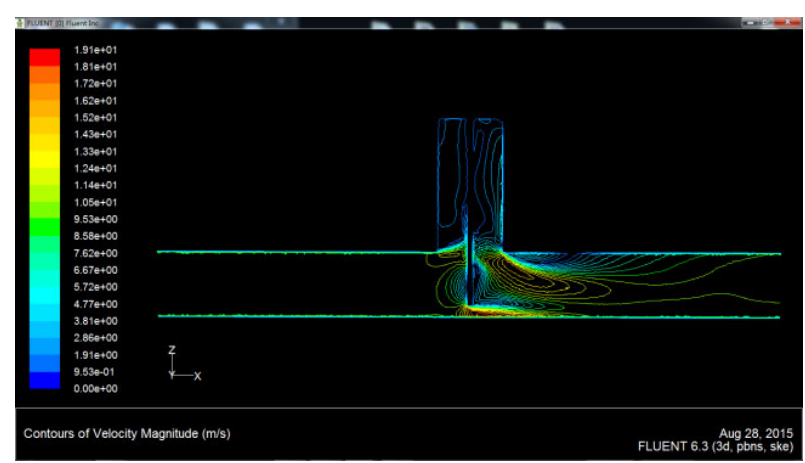

(a)

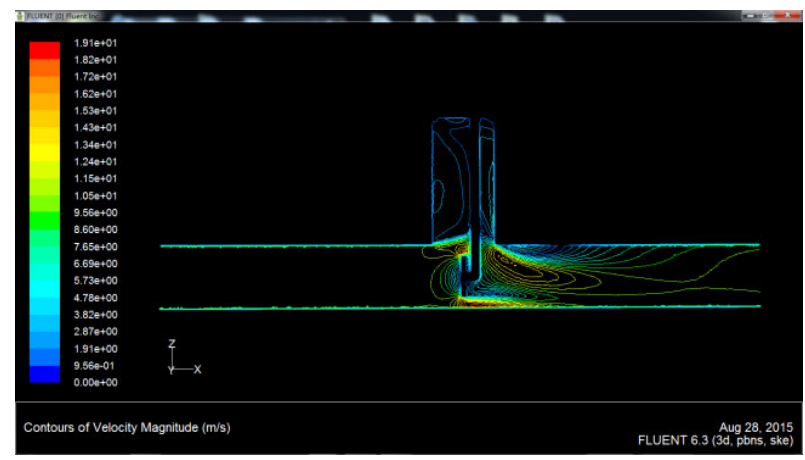

(b)

Fig. 6 Velocity isoline: (a) one-piece target type structure and (b) traditional model of the target structure.

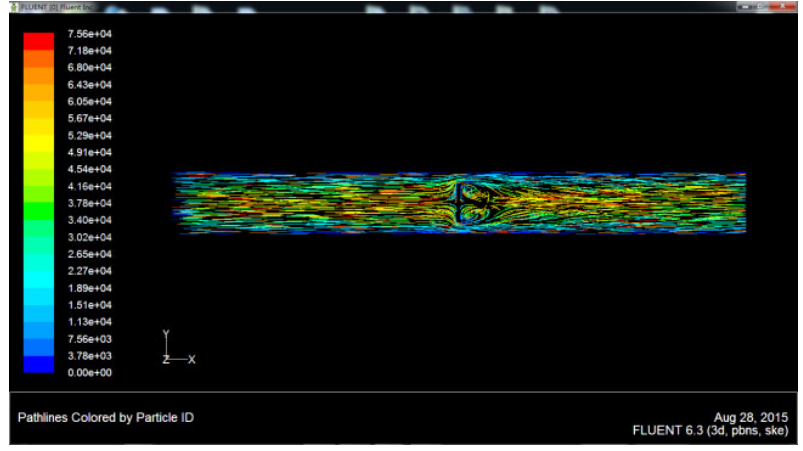

(a)

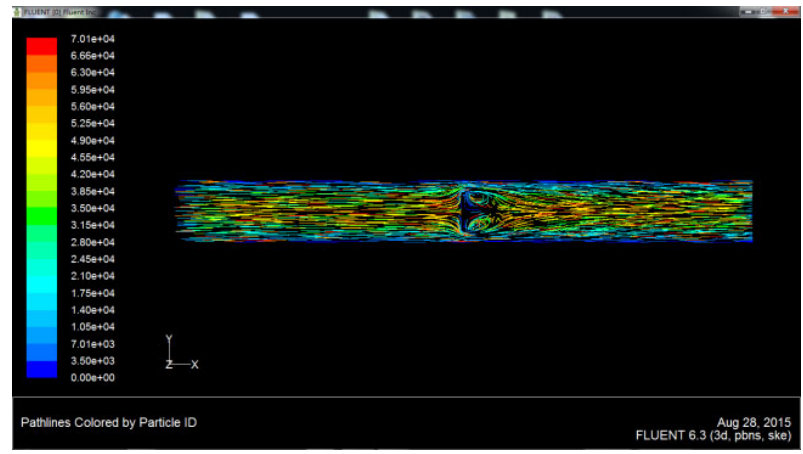

(b)

Fig. 7 Motion pattern: (a) one-piece target type structure and (b) traditional model of the target structure.

Through the above simulation results, it can be seen that with the same flow, one-piece flow sensor target type's influence on the fluid is smaller than that of the traditional target type flow sensor. After the fluid flows through the target piece, state of fluid can restore equilibrium within a short distance. Thus it can be seen that design of the structure of one-piece target type flow sensor is reasonable and feasible.

\section{FBG flow sensing experiment}

The hydraulic loop is built as shown in Fig. 8 in order to further verify the sensing properties of one-piece target type flow sensor based on FBG. Mobile oil source device is mainly composed of hydraulic pumps and fuel tanks and supplies oil for the system. The one-piece target type flow sensor is in the hydraulic circuit. A smart turbine flowmeter is in series as the standard of flow measurement and the flow of the circuit is adjusted by the throttle. The measuring range is between $0.5 \mathrm{~m}^{3} / \mathrm{h}$ and $10 \mathrm{~m}^{3} / \mathrm{h}$ that between $0.14 \mathrm{~L} / \mathrm{s}$ and $2.78 \mathrm{~L} / \mathrm{s}$, the measurement 
accuracy is 1.0 , the nominal diameter is $10 \mathrm{~mm}$, and the pressure limit is $25 \mathrm{MPa}$. In the connection of the system, there should be two pipes with the diameter of $8 \mathrm{D}$ and $5 \mathrm{D}$ on both sides of the target piece according to the requirements of the target flowmeter and two pipes with the diameter of 20D and $5 \mathrm{D}$ according to the requirements of the turbine flowmeter. The liquid can only flow through the turbine flowmeter in one way.

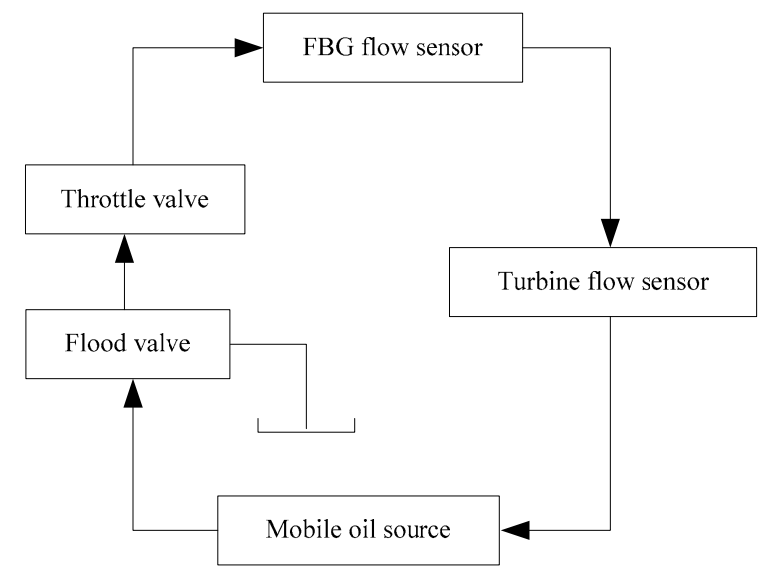

Fig. 8 Flow test system circuit diagram.

Figure 9 shows the assembled one-piece target type flow sensor, and two FBGs with the central wavelength $\lambda_{B}=1550 \mathrm{~nm}$ are pasted symmetrically on both sides of the central axis of the triangle cantilever beam by using the modified epoxy resin adhesives, and they are fused in series by using the optical fiber fusion splicer (OFFS). The optical fiber is between the plywood and pressure diaphragm, and FC plug is fused using the OFFS.

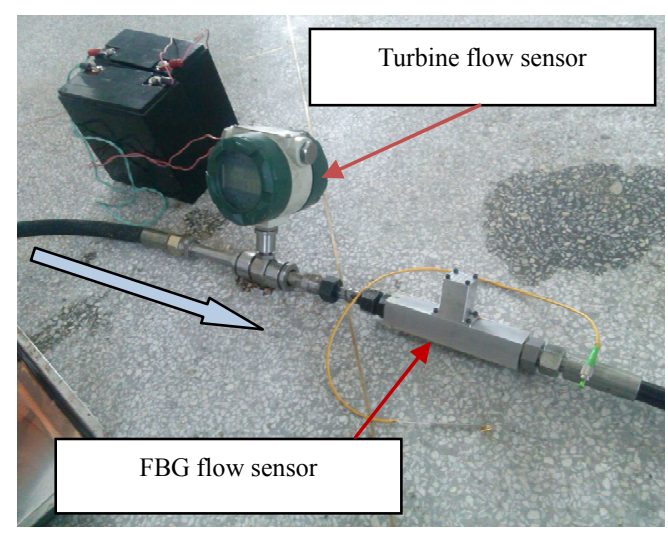

Fig. 9 Flow sensor test system experimental photos.
Fig. 10 shows the experimental photographs. In the hydraulic circuit of flow test, the oil is pumped out from the pump into the overflow valve, throttle valve, the flow testing equipment, the turbine flowmeter, the one-piece flow target type optical FBG sensor, and the tank at last.

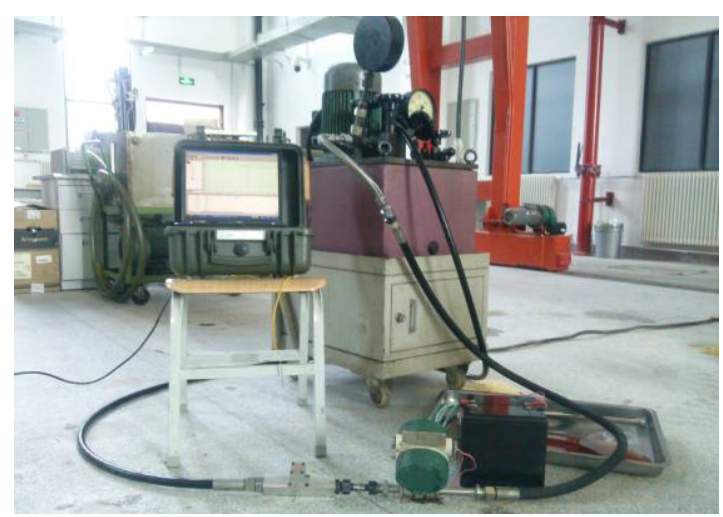

Fig. 10 Flow sensor test system experimental photos.

In the experiment, we connect the test oil and the sensing light path, start the oil source, read the central wavelength of a pair of FBG in the demodulation equipment as the experimental data when the flow is $0 \mathrm{~L} / \mathrm{s}$ and then start the oil source, adjust the opening size of the throttle valve to increase the flow gradually in the test loop, and record the central wavelength values of FBG1 and FBG2 target type flow sensor respectively every $0.2 \mathrm{~L} / \mathrm{s}$ after the system is stable. The test data is shown in Table 1.

Table 1 Flow sensing experiment.

\begin{tabular}{cccccc}
\hline \multirow{2}{*}{ Flow (L/s) } & \multicolumn{5}{c}{ Central wavelength (nm) } \\
\cline { 2 - 6 } & FBG1 & $\Delta \lambda_{B 1}$ & FBG2 & $\Delta \lambda_{B 2}$ & $\Delta \lambda_{B}$ \\
\hline 0 & 1550.083 & 0.083 & 1549.944 & 0.056 & 0.139 \\
0.2 & 1550.163 & 0.163 & 1549.862 & 0.138 & 0.301 \\
0.4 & 1550.227 & 0.227 & 1549.796 & 0.204 & 0.431 \\
0.6 & 1550.355 & 0.355 & 1549.672 & 0.328 & 0.683 \\
0.8 & 1550.474 & 0.474 & 1549.551 & 0.449 & 0.923 \\
1.0 & 1550.58 & 0.58 & 1549.447 & 0.553 & 1.133 \\
1.2 & 1550.761 & 0.761 & 1549.266 & 0.734 & 1.495 \\
1.4 & 1550.923 & 0.923 & 1549.101 & 0.899 & 1.822 \\
1.6 & 1551.105 & 1.105 & 1548.922 & 1.078 & 2.183 \\
1.8 & 1551.332 & 1.332 & 1548.688 & 1.312 & 2.644 \\
2.0 & 1551.507 & 1.507 & 1548.52 & 1.48 & 2.987 \\
2.2 & 1551.792 & 1.792 & 1548.227 & 1.773 & 3.565 \\
\hline
\end{tabular}


Figure 11 shows the offset of the central wavelength of FBG1, FBG2, and double FBG after fitting the experimental data.

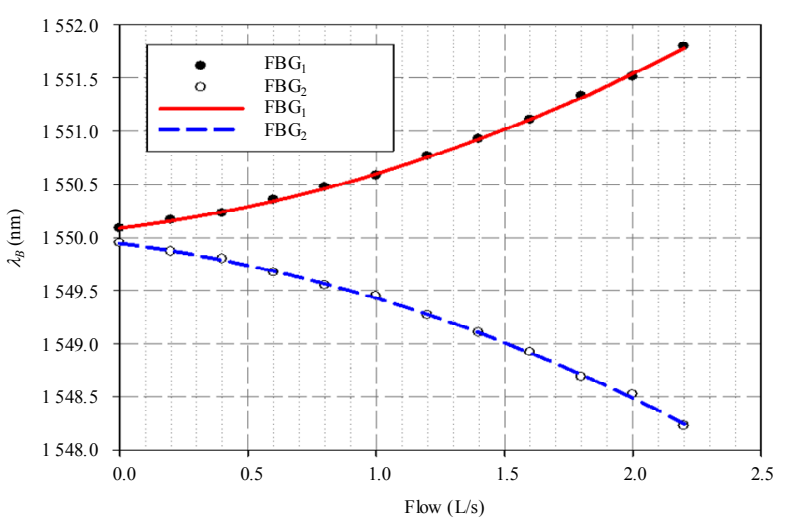

(a)

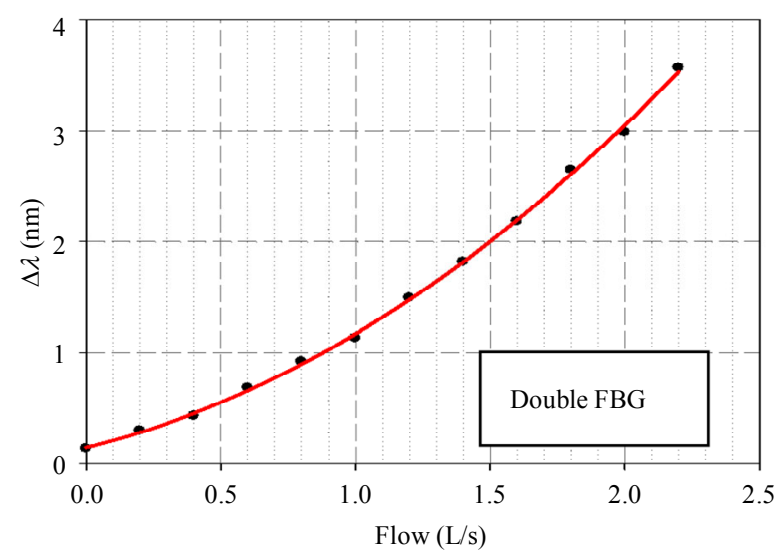

(b)

Fig. 11 Flow sensor experimental data fitting: (a) $\mathrm{FBG}_{1}$ and $\mathrm{FBG}_{2}$ central wavelength offset fitting processing and (b) double FBG wavelength offset fitting processing.

According to (10), the offset of the FBG central wavelength in the one-piece target type flow sensor is proportional to the square of the flow, and the test data after fitting are obviously quadratic, so the fitting curve equation is given as

$$
\left\{\begin{array}{l}
\lambda_{B 1}=1550.087+0.213 Q^{2} \\
\lambda_{B 2}=1549.938+0.216 Q^{2} \\
\Delta \lambda_{B}=0.1419+0.423 Q^{2} .
\end{array}\right.
$$

Fitting curves prove that the offset of the central wavelength of the sensor is proportional to the square of the flow. The offset of the central wavelength of double FBG is twice as large as that of one FBG, and the flow sensor sensitivity of the differential configuration of the double FBG is doubled. And we can get the resolution of the one-piece target type flow sensor is equal to $0.049 \mathrm{~L} / \mathrm{s}$ from that the quadratic term coefficient is equal to $0.423 \mathrm{~nm} /(\mathrm{L} / \mathrm{s})^{2}$, and the resolution of the demodulation instrument is equal to $1 \mathrm{pm}$.

\section{Conclusions}

In the paper, we design a construction which combines target sheet and sensitive element into one product based on the sensing principle of the FBG target type flowmeter. The power transmission structure is omitted, and the triangle cantilever beam is selected as the fastener and the force sensor of the target sheet. Two FBGs, of which the central wavelength of which are equal, are affixed on the central axis of both sides of the cantilever beam because of its sensitivity to the deformation of the cantilever beam caused by the forces on the target sheet. The FLUENT software is employed for the simulation analysis of the influence that one-piece target piece has on the fluid state and the conclusion is reached that the influence of the one-piece target piece structure on the fluid state is less than that of traditional structures. The feasibility and the reliability of the sensor design are verified through flow experiments, the sensor sensitivity is improved effectively, and the measurement repeatability of the sensor is excellent. The one-piece structure target flow sensor based on FBG is compact and convenient to be sealed, dismantled, and installed. What is more, it can adapt to the inflammables, explosion-prone objects, strong electromagnetic radiation, the long-distance test, and the distributed test, so it has broad application prospects.

\section{Acknowledgment}

This work was supported by the National Natural Science Foundation of China (No. 41404022) and the Shanxi National Science Foundation (No.2015JM4128). 
Open Access This article is distributed under the terms of the Creative Commons Attribution 4.0 International License (http://creativecommons.org/licenses/by/4.0/), which permits unrestricted use, distribution, and reproduction in any medium, provided you give appropriate credit to the original author(s) and the source, provide a link to the Creative Commons license, and indicate if changes were made.

\section{References}

[1] L. Z. Liang, Z. J. Chen, and Y. B. Wang, "Signal detector of propeller flow velocity meter," Process Automation Instrumentation, 2003, 24(5): 41-43.

[2] D. D. Zheng, P. Y. Zhang, and T. Zhang, "A method based on a novel flow pattern model for the flow adaptability study of ultrasonic flowmeter," Flow Measurement and Instrumentation, 2013, 29(1): 25-31.

[3] T. Leeungculsatien and G. P. Lucas, "Measurement of velocity profiles in multiphase flow using a multi-electrode electromagnetic fow meter," Flow Measurement and Instrumentation, 2013, 31(6): 86-95.

[4] C. L. Hu, Z. S. Lin, and Y. Y. Chen, "Portable laser Doppler flowmeter for microcirculation detection," Biomedical Engineering Letters, 2013, 3(2): 109-114.

[5] D. S. Wang, The study of flow measurement based on the optical fiber gating system, Ph.D. dissertation, Yanshan University, 2013.

[6] X. Z. Zhang and X. Y. Li, "Optical fiber flow sensor," Transducer Technology, 1999, 18(1): 57-59.

[7] M. Majiimder, T. K. Gangopadhyay, and A. K. Chakraborty, "Fiber Bragg gratings in structural health monitoring-present status and applications," Sensors and Actuators: A Physical, 2008, 147(1): 150-164.
[8] Y. B. Wang and H. J Lan, "Study of fiber Bragg grating sensor system based on wavelength-division multiplexing/time-devision mltiplexing," Acta Photonica Sinica, 2010, 30(8): 2196-2201.

[9] D. C. Robertson, P. Niewczas, and J. R. Mcdonald, "Interrogation of a dual-fiber-Bragg-grating sensor using an arrayed waveguide grating," IEEE Transactions on Instrumentation and Measurement, 2007, 56(6): 2641-2645.

[10] M. S. Jiang, Q. M. Sui, and L. Jia, "FBG-based ultrasonic wave detection and acoustic emission linear location system," Optoelectronics Letters, 2012, 8(3): 220-223.

[11] D. K. Yu, Z. A. Jia, and X. G. Qiao, "FBG sensors combining target with cantilever beam for simultaneous measurement of flow-rate/ temperature," Journal of Optoelectronics Laser, 2010, 21(5): 710-713.

[12] Z. F. Wang, J. Wang, and Q. M. Sui, "A target-type fiber Bragg grating flow velocity sensor," Journal of Optoelectronics Laser, 2012, 23(1): 1-8.

[13] D. S. Wang, Y. T. Wang, and W. W. Pan, "A circular target flow measurement system," Applied Mechanics and Materials, 2013, 427-429: 1233-1236.

[14] H. C. Li, C. T. Liu, and Y. B. Feng, "An embedded nozzle differential pressure style flow sensor based on FBG," Journal of Optoelectronics Laser, 2014, 25(10): 1886-1891.

[15] S. Takashima, H. Asanuma, and H. Niitsuma, "A water flowmeter using dual fiber Bragg grating sensors and cross-correlation technique," Sensors and Actuarors A: Physical, 2004, 116(1): 66-74.

[16] Y. Zhao, K. Chen, and J. Yang, "Novel target type flowmeter based on a differential fiber Bragg grating sensor," Measurement, 2005, 38(3): 230-235.

[17] Y. R. Hu, Y. H. Tian, and C. Li, "Fiber Bragg grating flow sensor," Chinese Journal of Sensors and Actuators, 2010, 23(4): 471-474. 